(C2014 IEEE. Personal use of this material is permitted. Permission from IEEE must be obtained for all other uses, in any current or future media, including reprinting/republishing this material for advertising or promotional purposes, creating new collective works, for resale or redistribution to servers or lists, or reuse of any copyrighted component of this work in other works. This is the author's version of an article that has been published in the conference proceedings. The final version of record is available at doi: http://dx.doi.org/10.1109/PLANS.2016.7479776 


\title{
Simultaneous Localization and Mapping in Multipath Environments
}

\author{
Christian Gentner, Boxiao Ma, Markus Ulmschneider, Thomas Jost, Armin Dammann \\ German Aerospace Center (DLR) \\ Institute of Communications and Navigation \\ Oberpfaffenhofen, 82234 Wessling, Germany \\ Email: \{Christian.Gentner, Boxiao.Ma, Markus.Ulmschneider, Thomas.Jost, Armin.Dammann\}@dlr.de
}

\begin{abstract}
This paper presents and extends the idea of multipath assisted positioning, named Channel-SLAM. Generally, multipath reception degrades the accuracy of the positioning device as long as the receiver is based on standard methods. In contrast, Channel-SLAM uses the multipath propagation of the wireless signal to allow positioning in cases of insufficient number of transmitters or increase the accuracy otherwise. Channel-SLAM treats multipath components (MPCs) as signals from virtual transmitters (VTs) which are time synchronized to the physical transmitter and fixed in their position. To use the information of the MPCs, Channel-SLAM estimates the receiver position and the position of the VTs simultaneously and does not require any prior information such as room-layout or a database for fingerprinting. The simultaneous localization and mapping (SLAM) algorithm is used by the receiver to estimate its own position and the position of VTs as landmarks. This paper investigates mapping of the receiver position, where we derive a probabilistic map representation based on locations. Thus, if the receiver knows its current location, we also know the probability where the receiver moves for the next step. In order to estimate and store the probability distribution of receivers motions as a function of location, we propose a probabilistic map that represents the receiver motion in a two-dimensional hexagonal grid. Hence, as soon as the receiver returns to an already mapped position, information of this position can be reused for positioning to obtain better position estimations of the receiver position. The algorithm is evaluated based on measurements with one fixed transmitter and a moving pedestrian which moves on partially overlapping loops. Based on these evaluations, we show, that the algorithm is able to accurately map the trajectory as well as reuse estimated map.
\end{abstract}

\section{INTRODUCTION}

With Channel-SLAM we introduced in [1]-[4] a novel algorithm which uses MPCs for positioning instead of mitigating them. Measurements with a moving receive antenna showed, that some MPCs have a path life of several meters of the receiver movement. These long visible paths can be used by Channel-SLAM for positioning. Hence, Channel-SLAM treats each MPC as a line-of-sight signal from a VT which position is unknown to the receiver. These VTs are static during the receiver movement. Channel-SLAM estimates the receiver position and the positions of the VTs simultaneously, thus, contrarily to other approaches like [5]-[8] the approach does not require any prior information such as room-layout or a database for fingerprinting. The only three conditions to be fulfilled are the presence of a multipath environment, a moving receiver as well as an initial prior knowledge of the receiver states, i.e. position and movement.

This work builds on and extends the previous work on Channel-SLAM. Channel-SLAM basically uses a two level approach: The first level uses Kalman enhanced super resolution tracking (KEST) [9] to estimate and track the amplitude and the delay of each MPC. The second level estimates the positions of the receiver and the VTs based on the estimated parameters of the MPCs simultaneously. Due to the movement of the receiver, we use a recursive Bayesian filter approach to estimate the probability density function of the receiver position and the VT positions. Recently, we extended ChannelSLAM for pedestrians and fused the heading estimations of a gyroscope with Channel-SLAM. We evaluated the algorithm based on measurements with one transmitter and a pedestrian carrying the receive antenna and a gyroscope. These evaluations demonstrated the concept of Channel-SLAM and showed that Channel-SLAM is able to determine VT positions accurately. A method to estimate the position of the receiver at the same time as landmarks is called SLAM, see [10]. Here, the SLAM algorithm is used by the receiver to estimate its own position and the position of VTs as landmarks. Usually in robotics, SLAM covers the task of asserting whether the robot returned to a previously visited area, after moving for an arbitrary time which is called loop closure. This allows the robot to reuse previously gained information. Hence, we derive in this paper a probabilistic map representation based on locations. Thus, if the receiver knows its current location, we know the probability where the receiver will move for the next step. In order to estimate and store the probability distribution of receivers motions as a function of location, we need to partition the space. We propose a probabilistic map that represents the receiver motion in a two-dimensional hexagonal grid. Hence, as soon as the receiver returns to an already mapped position, information of this position can be reused for positioning which obtains better position estimations of the receiver position. To verify the refined algorithm, we perform evaluations based on measurements. We used a scenario with one fixed transmitter and a moving pedestrian, carrying the receive antenna and a gyroscope in his hands. The pedestrian was moving on partly overlapping loops. Hence, we show that the algorithm is able to map the trajectory as well as reuse the estimated map. 
The paper is structured as follows: Section II describes the signal model and VTs, afterwards Section III derives the proposed algorithm which is split into three subsections: Section III-A addresses Channel-SLAM; Section III-B addresses the mapping of the receiver position; Section III-C addresses the particle filter $(\mathrm{PF})$ implementation with the weight calculation; Thereafter, Section IV evaluates the algorithm based on measurements. The last section, Section V, concludes the paper.

Throughout the paper, we will use the following notation:

- $(\cdot)^{T},(\cdot)^{H}$ stands for matrix (or vector) transpose and conjugate transpose, respectively.

- All vectors are interpreted as column vectors.

- $\mathbf{x} \odot \mathbf{y}$ denotes the Hadamard-Schur product, i.e. the element-wise multiplication of vector $\mathbf{x}$ and $\mathbf{y}$.

- I denotes an identity matrix.

- Matrices are denoted by bold capital letters and vectors by bold small letters.

- $\|\mathbf{A}\|^{2}=\sum_{l} \sum_{m}\left|A_{l, m}\right|^{2}$ represents the square of the Frobenius norm of $\mathbf{A}$ with elements $A_{l, m}$.

- $a \sim \mathcal{N}\left(x ; \mu_{a}, \sigma_{a}^{2}\right)$ denotes a Gaussian distributed random variable $a$ with mean $\mu_{a}$ and variance $\sigma_{a}^{2}$.

- $1: k$ stands for all integer numbers starting from 1 to $k$, thus $1,2, \ldots, k$.

- $\mathrm{p}(x)$ denotes the probability density function of $x$.

- $f^{-1}(\cdot)$ denotes the inverse of function $f(\cdot)$.

- $c$ is the speed of light.

\section{Signal MODEL}

As mentioned in the introduction, with Channel-SLAM [1][4] we introduced a novel algorithm which uses MPCs for positioning instead of mitigating them. Measurements with a moving receive antenna showed, that some MPCs have a path life of several meters of the receiver movement [11]. These long visible paths can be used by Channel-SLAM for positioning. Fig. 1 shows a scenario, where the transmitted signal is reflected on a smooth surface. The transmitter has a fixed position and the receiver is moving on the indicated path. When the receiver is moving also the reflection point, indicated by $R\left(t_{k}\right)$ at the coordinates $\boldsymbol{r}_{r}\left(t_{k}\right)$ is moving. If we mirror the physical transmitter position on the reflecting surface, we obtain the position $\boldsymbol{r}_{\mathrm{VT}}$ of the VT which is static during the receiver movement. The distance between the VT and the receiver is equal to $d_{\mathrm{TR}}\left(t_{k}\right)+d_{\mathrm{RU}}\left(t_{k}\right)=$ $\left\|\boldsymbol{r}_{t}-\boldsymbol{r}_{r}\left(t_{k}\right)\right\|+\left\|\boldsymbol{r}_{r}\left(t_{k}\right)-\boldsymbol{r}_{u}\left(t_{k}\right)\right\|=\left\|\boldsymbol{r}_{\mathrm{VT}}-\boldsymbol{r}_{u}\left(t_{k}\right)\right\|$ which is equivalent to the propagation time of the reflected signal multiplied with the speed of light. Fig. 1 exploits additionally a scenario where the signal is scattered. The propagation effect of scattering occurs if an electromagnetic wave impinges an object and the energy is spread out in all directions. Geometrically, the effect of scattering can be described as a fixed point $S$ in the pathway of the MPC for all receiver positions. Hence, the propagation distance of the scattered path is $d_{\mathrm{TS}}\left(t_{k}\right)+d_{\mathrm{SU}}\left(t_{k}\right)=\left\|\boldsymbol{r}_{t}-\boldsymbol{r}_{s}\right\|+\left\|\boldsymbol{r}_{s}-\boldsymbol{r}_{u}\left(t_{k}\right)\right\|=$ $\left\|\boldsymbol{r}_{\mathrm{VT}}-\boldsymbol{r}_{u}\left(t_{k}\right)\right\|+d_{\mathrm{VT}}$ where $d_{\mathrm{TS}}\left(t_{k}\right)=d_{\mathrm{VT}}>0$ is constant and $\boldsymbol{r}_{s}=\boldsymbol{r}_{\mathrm{VT}}$ denotes the position of the scatterer. Thus,

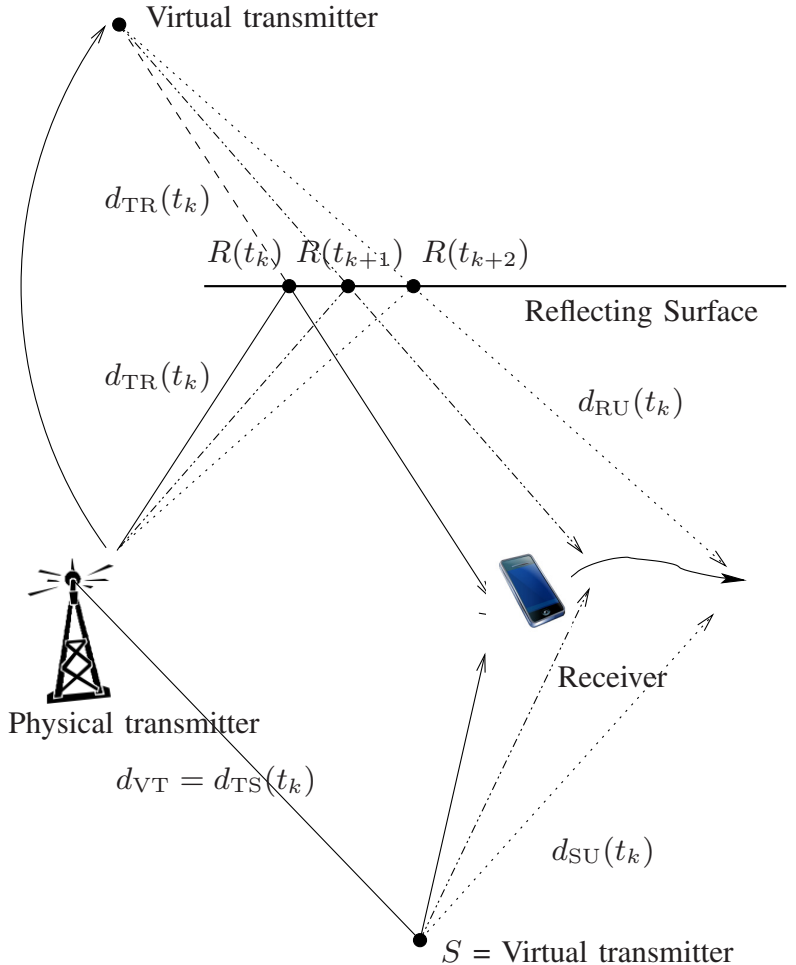

Fig. 1: Two Scenarios: First: The transmitted signal is reflected at a smooth surface. When the receiver is moving the reflection point $R\left(t_{k}\right)$ is moving on the surface, indicated by $R\left(t_{k}\right)$, $R\left(t_{k+1}\right)$ and $R\left(t_{k+2}\right)$. A VT can be defined by mirroring the physical transmitter position at the surface as visualized. Second: signal is scattered at $S$. When the receiver is moving, $S$ is fixed, hence $S$ is defined as the position of VT. For the propagation length a constant $d_{\mathrm{VT}}$ needs to be added.

we define $S$ as VT for the MPC and treat $d_{\mathrm{VT}}>0$ as an additional propagation distance associated to the MPC. Fig. 2 shows a generalization of the considered multipath effects. The transmitted signal is scattered at $S$ and afterwards reflected. Between the transmitter and $S$ additional interactions are possible. When the receiver is moving, the reflection point $R\left(t_{k}\right)$ is moving on the surface. Hence, the VT is defined by mirroring the scatterer $S$ at the surface as visualized. The propagation distance is therefore $d_{\mathrm{TS}}\left(t_{k}\right)+d_{\mathrm{SR}}\left(t_{k}\right)+$ $d_{\mathrm{RU}}\left(t_{k}\right)=d_{\mathrm{VT}}+\left\|\boldsymbol{r}_{s}\left(t_{k}\right)-\boldsymbol{r}_{r}\left(t_{k}\right)\right\|+\left\|\boldsymbol{r}_{r}\left(t_{k}\right)-\boldsymbol{r}_{u}\left(t_{k}\right)\right\|=$ $d_{\mathrm{VT}}+\left\|\boldsymbol{r}_{\mathrm{VT}}-\boldsymbol{r}_{u}\left(t_{k}\right)\right\|$ where $d_{\mathrm{TS}}\left(t_{k}\right)=d_{\mathrm{VT}}>0$ is constant.

To summarize, the propagation path of each MPC can be equivalently described as a direct path between a VT and the receiver plus an additional constant propagation length $d_{\mathrm{VT}}$. If only reflections occurred on the pathway between physical transmitter and receiver, this additional propagation length is zero $d_{\mathrm{VT}}=0$. If the MPC was interacting with a scatterer, the additional propagation length is greater than zero $d_{\mathrm{VT}}>0$. Equivalently, we can interpret $d_{\mathrm{VT}}$ as a constant clock offset between the VT and the physical transmitter. Hence, ChannelSLAM treats each MPC as a line-of-sight signal from a VT 


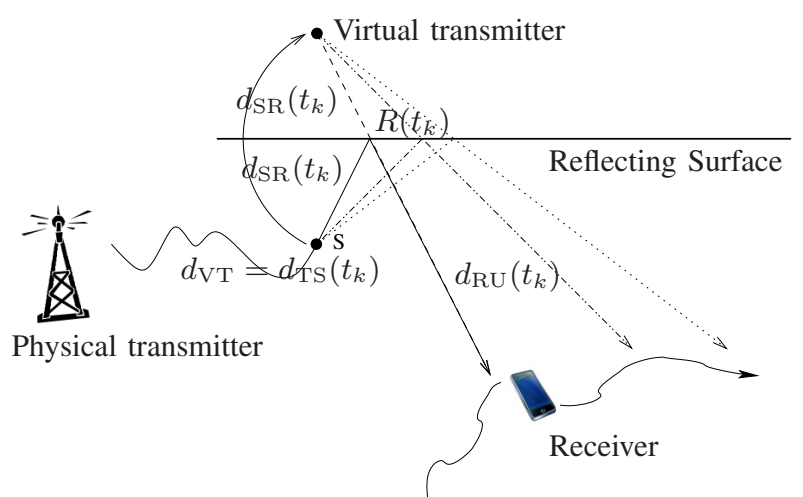

Fig. 2: The transmitted signal is scattered and afterwards reflected at a smooth surface. When the receiver is moving the reflection point $R\left(t_{k}\right)$ is moving on the surface. A VT can be defined by mirroring the scatterer $S$ at the surface as visualized. For the propagation length a constant $d_{\mathrm{VT}}$ needs to be added.

whose position is unknown to the receiver. These VTs are static during the receiver movement.

\section{Multipath Assisted Positioning}

As mentioned in [1]-[4], the proposed algorithm considers a multi level approach. KEST estimates and tracks the channel impulse responses (CIRs) between $N_{\mathrm{T}}$ physical transmitters and the receiver. We assume that the signals transmitted by each physical transmitter are separable, i.e. orthogonal in the ideal case. Hence, the received signal vectors $\boldsymbol{y}_{v}\left(t_{k}\right)$ of transmitter $v=1 \ldots N_{\mathrm{T}}{ }^{1}$ are processed by the KEST algorithm, which estimates for each MPC $i$, the propagation distance $d_{v, i}\left(t_{k}\right)=\tau_{v, i}\left(t_{k}\right) \cdot c$. For each transmitter $v=1, \ldots, N_{\mathrm{T}}$ we define the vector $\tilde{\boldsymbol{d}}_{v}\left(t_{k}\right)$ with

$$
\tilde{\boldsymbol{d}}_{v}\left(t_{k}\right)=\left[\tilde{d}_{v, 0}\left(t_{k}\right), \ldots, \tilde{d}_{v, N_{v}\left(t_{k}\right)-1}\left(t_{k}\right)\right]^{T}
$$

with the KEST estimates for $d_{v, i}\left(t_{k}\right)$. The measurement vector $\mathbf{z}\left(t_{k}\right)$ for Channel-SLAM is defined as

$$
\mathbf{z}\left(t_{k}\right)=\left[\tilde{\boldsymbol{d}}_{1}\left(t_{k}\right)^{T}, \ldots, \tilde{\boldsymbol{d}}_{N_{\mathrm{T}}}\left(t_{k}\right)^{T}\right]^{T} .
$$

For each MPC $i$ of transmitter $v=1, \ldots, N_{\mathrm{T}}$, with $i=0, \ldots, N_{v}\left(t_{k}\right)-1$, we can define a VT with its position $\boldsymbol{r}_{\mathrm{VT}, v, i}\left(t_{k}\right)$ and an additional propagation length $d_{\mathrm{VT}, v, i}\left(t_{k}\right)$. Knowing the positions of the VTs, the receiver position $\boldsymbol{r}_{u}\left(t_{k}\right)$ for time instant $t_{k}$ can be estimated. A method to estimate the position of the receiver at the same time as landmarks is called SLAM, see [10]. Here, the SLAM algorithm is used by the receiver to estimate its own position and the position of VTs as landmarks. Usually, SLAM covers the task of asserting whether the receiver returned to a previously visited area, after moving for arbitrary time called loop closure. Realizing reliable loop closure is both essential and challenging, which is without doubt one of the greatest impediments to achieve longterm and robust SLAM. Hence, in a Bayesian formulation, we

\footnotetext{
${ }^{1}$ Please note, that we will set $N_{\mathrm{T}}=1$ in Section IV.
}

are interested in the posterior

$$
\mathrm{p}\left(\mathbf{x}\left(t_{0: k}\right), \mathbf{M}\left(t_{0: k}\right) \mid \mathbf{z}\left(t_{0: k}\right)\right)
$$

where $\mathbf{M}\left(t_{0: k}\right)$ defines the probability map of receivers motion. We can factorize (3) into

$$
\begin{aligned}
\mathbf{p} & \left(\mathbf{x}\left(t_{0: k}\right), \mathbf{M}\left(t_{0: k}\right) \mid \mathbf{z}\left(t_{0: k}\right)\right) \\
& =\mathbf{p}\left(\mathbf{x}\left(t_{0: k}\right) \mid \mathbf{z}\left(t_{0: k}\right)\right) \cdot \mathbf{p}\left(\mathbf{M}\left(t_{0: k}\right) \mid \mathbf{z}\left(t_{0: k}\right), \mathbf{x}\left(t_{0: k}\right)\right) \\
& =\underbrace{\mathbf{p}\left(\mathbf{x}\left(t_{0: k}\right) \mid \mathbf{z}\left(t_{0: k}\right)\right)}_{\text {Channel-SLAM }} \cdot \underbrace{\mathbf{p}\left(\mathbf{M}\left(t_{0: k}\right) \mid \mathbf{x}_{u}\left(t_{0: k}\right)\right)}_{\text {mapping problem conditioned on the receiver state }}
\end{aligned}
$$

where we assume that the map only depends on the receiver state $\mathbf{x}_{u}\left(t_{k}\right)$. In (4), the first term $\mathrm{p}\left(\mathbf{x}\left(t_{0: k}\right) \mid \mathbf{z}\left(t_{0: k}\right)\right)$ defines the posterior of Channel-SLAM described in Section III-A and the second term $\mathrm{p}\left(\mathbf{M}\left(t_{0: k}\right) \mid \mathbf{x}_{u}\left(t_{0: k}\right)\right)$ defines the posterior of the mapping of the receiver described in Section III-B.

\section{A. Channel-SLAM}

Due to the movement of the receiver a tracking filter can be applied which recursively estimates the position of the receiver and of the landmarks. Recursive Bayesian filters estimate an unknown probability density function (PDF) over time using measurements and mathematical defined models, see [12]. Thus, recursive Bayesian filters can be used to estimate the PDF of a state vector $\mathbf{x}\left(t_{k}\right)$ at time instant $t_{k}$ using the measurements $\mathbf{z}\left(t_{0: k}\right)$ from time instant $t_{0}$ to $t_{k}$. Hence, the posterior PDF $p\left(\mathbf{x}\left(t_{k}\right) \mid \mathbf{z}\left(t_{0: k}\right)\right)$ is estimated based on the prior estimate $p\left(\mathbf{x}\left(t_{k-1}\right) \mid \mathbf{z}\left(t_{0: k-1}\right)\right)$. Here, the state vector $\mathbf{x}\left(t_{k}\right)$ at time instant $t_{k}$ for $N_{v}\left(t_{k}\right)$ MPCs and $N_{\mathrm{T}}$ transmitters is defined by

$$
\mathbf{x}\left(t_{k}\right)=\left[\mathbf{x}_{u}\left(t_{k}\right)^{T}, \tilde{\mathbf{x}}_{\mathrm{VT}, 1}\left(t_{k}\right)^{T}, \ldots, \tilde{\mathbf{x}}_{\mathrm{VT}, N_{\mathrm{T}}}\left(t_{k}\right)^{T}\right]^{T},
$$

with the receiver state $\mathbf{x}_{u}\left(t_{k}\right)$ and with

$$
\tilde{\mathbf{x}}_{\mathrm{VT}, v}\left(t_{k}\right)=\left[\mathbf{x}_{\mathrm{VT}, v, 0}\left(t_{k}\right)^{T}, \ldots, \mathbf{x}_{\mathrm{VT}, 0, N_{v}\left(t_{k}\right)-1}\left(t_{k}\right)^{T}\right]^{T},
$$

for each transmitter $v=1 \ldots N_{\mathrm{T}}$ and

$$
\mathbf{x}_{u}\left(t_{k}\right)=\left[\boldsymbol{r}_{u}\left(t_{k}\right)^{T}, \boldsymbol{v}_{u}\left(t_{k}\right)^{T}, b_{u}\left(t_{k}\right), \rho_{u}\left(t_{k}\right)\right]^{T},
$$

where $\boldsymbol{r}_{u}\left(t_{k}\right)$ is the receiver position, $\boldsymbol{v}_{u}\left(t_{k}\right)$ the receiver velocity, $b_{u}\left(t_{k}\right)$ and $\rho_{u}\left(t_{k}\right)$ the receiver's clock bias and drift, respectively. The parameters representing the VT of the $i$-th MPC of transmitter $v$ are defined as

$$
\mathbf{x}_{\mathrm{VT}, v, i}\left(t_{k}\right)=\left[\boldsymbol{r}_{\mathrm{VT}, v, i}\left(t_{k}\right)^{T}, d_{\mathrm{VT}, v, i}\left(t_{k}\right)\right]^{T},
$$

where $\boldsymbol{r}_{\mathrm{VT}, v, i}\left(t_{k}\right)$ are the coordinates of the VT and $d_{\mathrm{VT}, v, i}\left(t_{k}\right)$ the additional propagation distance.

Recursive Bayesian filtering consists of two steps, the prediction step $\mathrm{p}\left(\mathbf{x}\left(t_{k}\right) \mid \mathbf{z}\left(t_{0: k-1}\right)\right)$ and a so called update step with $\mathrm{p}\left(\mathbf{x}\left(t_{k}\right) \mid \mathbf{z}\left(t_{0: k}\right)\right)$, which includes the measurements $\mathbf{z}\left(t_{k}\right)$ at time instant $t_{k}$ via the likelihood density function $\mathrm{p}\left(\mathbf{z}\left(t_{k}\right) \mid \mathbf{x}\left(t_{k}\right)\right)$. Assuming a first-order Markov model, the transition prior $\mathrm{p}\left(\mathbf{x}\left(t_{k}\right) \mid \mathbf{x}\left(t_{k-1}\right)\right)$ used in the prediction step 
of the recursive Bayesian filter is defined here as

$$
\begin{aligned}
& \mathrm{p}\left(\mathbf{x}\left(t_{k}\right) \mid \mathbf{x}\left(t_{k-1}\right)\right) \\
& =\mathrm{p}\left(\mathbf{x}_{u}\left(t_{k}\right) \mid \mathbf{x}_{u}\left(t_{k-1}\right)\right) \\
& \quad \times \prod_{v=1}^{N_{\mathrm{T}}} \prod_{i=0}^{N_{v}\left(t_{k}\right)-1} \mathrm{p}\left(\mathbf{x}_{\mathrm{VT}, v, i}\left(t_{k}\right) \mid \mathbf{x}_{\mathrm{VT}, v, i}\left(t_{k-1}\right)\right)
\end{aligned}
$$

As described in the previous section, the transition prior PDF of the VT state vectors $\mathbf{x}_{\mathrm{VT}, v, i}\left(t_{k}\right)$, $\mathrm{p}\left(\mathbf{x}_{\mathrm{VT}, v, i}\left(t_{k}\right) \mid \mathbf{x}_{\mathrm{VT}, v, i}\left(t_{k-1}\right)\right)$ associated to the transmitters $v=1, \ldots, N_{\mathrm{T}}$ and the MPCs $i=0, \ldots, N_{v}\left(t_{k}\right)-1$ is stationary, hence,

$$
\mathbf{x}_{\mathrm{VT}, v, i}\left(t_{k-1}\right)=\mathbf{x}_{\mathrm{VT}, v, i}\left(t_{k}\right) .
$$

Additional to the radio signals, we use an inertial measurement unit (IMU) which provides heading information to the algorithm. The gyroscope provides angular rates $\boldsymbol{\Delta}\left(t_{k}\right)$ in $\circ / \mathrm{s}$, where we consider only the measurements $\Delta_{y, \beta}\left(t_{k}\right)$ in yaw direction, see [13], [14]. Obviously, to obtain the moving direction, angular rates $\Delta_{y, \beta}\left(t_{k}\right)$ have to be integrated over time. However, because the error accumulates during integration, we use the angular rates $\Delta_{y, \beta}\left(t_{k}\right)$, with

$$
\Delta_{\beta}\left(t_{k}\right)=\Delta_{y, \beta}\left(t_{k}\right)+n_{\beta}\left(t_{k}\right),
$$

where $n_{\beta}\left(t_{k}\right)$ is the heading noise using a von Mises distribution. Hence, for the transition prior PDF of the receiver state $\mathbf{x}_{u}\left(t_{k}\right), \mathrm{p}\left(\mathbf{x}_{u}\left(t_{k}\right) \mid \mathbf{x}_{u}\left(t_{k-1}\right)\right)$ in a two dimensional Cartesian coordinate system, the receiver position $\boldsymbol{r}_{u}\left(t_{k}\right)$ is calculated as

$$
\boldsymbol{r}_{u}\left(t_{k}\right)=\boldsymbol{r}_{u}\left(t_{k-1}\right)+\left(t_{k}-t_{k-1}\right) \boldsymbol{v}_{u}\left(t_{k}\right),
$$

with the receiver velocity

$$
\boldsymbol{v}_{u}\left(t_{k}\right)=\mathbf{R}\left(\Delta_{\beta}\left(t_{k}\right)\right) \cdot \boldsymbol{v}_{u}\left(t_{k-1}\right)+\mathbf{n}_{u}\left(t_{k}\right)
$$

and the rotation matrix

$$
\mathbf{R}\left(\Delta_{\beta}\left(t_{k}\right)\right)=\left(\begin{array}{cc}
\cos \left(\Delta_{\beta}\left(t_{k}\right)\right) & -\sin \left(\Delta_{\beta}\left(t_{k}\right)\right) \\
\sin \left(\Delta_{\beta}\left(t_{k}\right)\right) & \cos \left(\Delta_{\beta}\left(t_{k}\right)\right)
\end{array}\right),
$$

$\mathbf{n}_{u}\left(t_{k}\right) \sim \mathcal{N}\left(0, \mathbf{Q}_{u}\left(t_{k}\right)\right)$ is transition noise. For the clock bias and clock drift known prediction models can be applied, see e.g. [15], [16].

Assuming the elements of the vector $\mathbf{z}\left(t_{k}\right)$ to be independent Gaussian distributed, the likelihood PDF $\mathrm{p}\left(\mathbf{z}\left(t_{k}\right) \mid \mathbf{x}\left(t_{k}\right)\right)$ can be expressed as

$$
\begin{aligned}
& \mathrm{p}\left(\mathbf{z}\left(t_{k}\right) \mid \mathbf{x}\left(t_{k}\right)\right) \\
& \quad=\prod_{v=1}^{N_{\mathrm{T}}} \prod_{i=0}^{N_{v}\left(t_{k}\right)-1} \frac{1}{\sqrt{2 \pi} \sigma_{d, v, i}\left(t_{k}\right)} e^{-\frac{\left(\hat{d}_{v, i}\left(t_{k}\right)-d_{v, i}\left(t_{k}\right)\right)^{2}}{2 \sigma_{d, v, i}^{2}\left(t_{k}\right)}},
\end{aligned}
$$

where

$$
d_{v, i}\left(t_{k}\right)=\left\|\boldsymbol{r}_{u}\left(t_{k}\right)-\boldsymbol{r}_{\mathrm{VT}, v, i}\left(t_{k}\right)\right\|+d_{\mathrm{VT}, v, i}\left(t_{k}\right)+b_{u}\left(t_{k}\right) \cdot c
$$

for the MPC $i$ of transmitter $v$ where $\sigma_{d, v, i}^{2}$ denotes the variance.

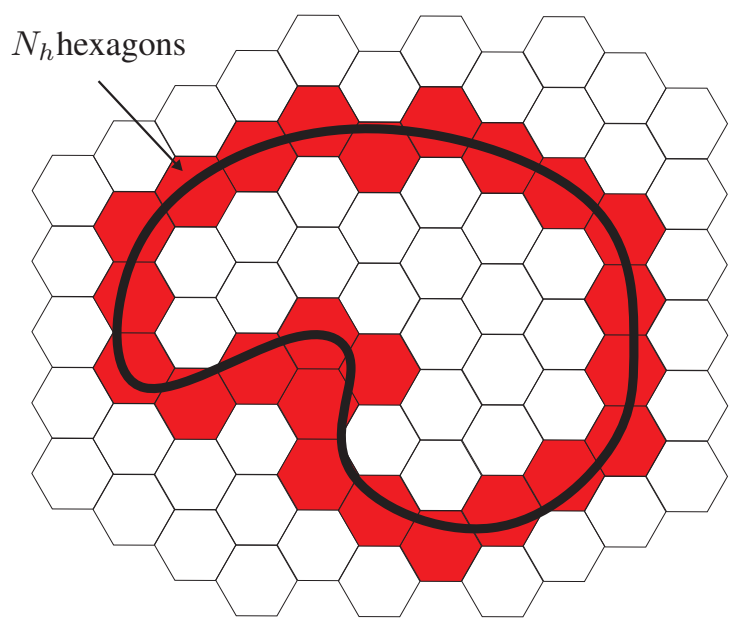

Fig. 3: Representing a walked path by hexagons: the black line indicates the walked path, the red hexagons shows the mapped path.

\section{B. Map generation}

To obtain $\mathrm{p}\left(\mathbf{M}\left(t_{0: k}\right) \mid \mathbf{x}_{u}\left(t_{0: k}\right)\right)$, we generate a probabilistic map based on locations. Thus, if we know the current location, we also know the probability where we move for the next step. In order to estimate and store the probability distribution of receivers motions as a function of location, we need to partition the space. Equivalently to [17]-[19], we discretize the space into a grid of adjacent and uniform hexagons. Fig. 3 shows an example where the walked path is indicated by the black line, the corresponding discretized hexagon map is indicated by $N_{h}$ hexagons indicated in red with $H=\left\{H_{0}, H_{1}, \ldots H_{N_{h}-1}\right\}$. Let us assume the receiver moved from hexagon $H_{i}$ to hexagon $H_{j}$ through the edge $e_{i, j}$ as shown in Fig. 4. Thus, we can define the transition probability of the crossed edge with

$$
m_{i}^{e}\left(t_{k}\right)=\mathrm{p}\left(\mathbf{x}_{u}\left(t_{k}\right) \in H_{j} \mid \mathbf{x}_{u}\left(t_{k-1}\right) \in H_{i}\right) .
$$

where $i \neq j$ and $e$ represents the index of each edge of the hexagon with $e=\{0,1, \ldots 5\}$ and $\sum_{e=0}^{5} m_{i}^{e}=1$. We denote with $\mathbf{m}_{i}^{e}\left(t_{k}\right)$ the map random variable, a probability, that is unknown to us. By observing the receiver states $\mathbf{x}_{u}\left(t_{0: k}\right)$ we can estimate $\mathrm{p}\left(\mathbf{m}_{i}^{e}\left(t_{k}\right) \mid \mathbf{x}_{u}\left(t_{0: k}\right)\right)$.

The map random variable $\mathbf{M}\left(t_{k}\right)$ can be decomposed as

$$
\mathbf{M}\left(t_{k}\right)=\left\{\mathbf{M}_{0}, \mathbf{M}_{1}, \ldots, \mathbf{M}_{i}, \ldots, \mathbf{M}_{N_{h}-1}\right\}
$$

where $\mathbf{M}_{i}$ is a random variable vector of length 6 denoting the transition probabilities of the hexagon with index $i$. The division of the space into independent hexagons makes the decomposition of the mapping problem into map estimation sub-problems possible [17], one for each hexagon, with

$$
\mathrm{p}\left(\mathbf{M}\left(t_{k}\right) \mid \mathbf{x}_{u}\left(t_{0: k}\right)\right)=\prod_{j=0}^{N_{h}-1} \mathrm{p}\left(\mathbf{M}_{j} \mid \mathbf{x}_{u}\left(t_{0: k}\right)\right) .
$$

Please note, for notational conveniences $\mathbf{M}_{i}$ do not depend on the time $t_{k}$ because the receiver might stay in one hexagon for more than one time step. 


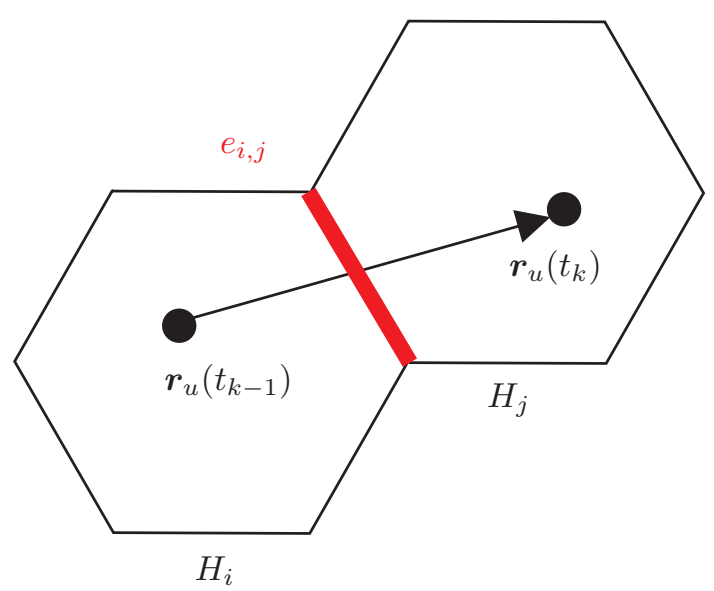

Fig. 4: Receiver movement form $\boldsymbol{r}_{u}\left(t_{k-1}\right)$ to $\boldsymbol{r}_{u}\left(t_{k}\right)$, respectively from hexagon $H_{i}$ to hexagon $H_{j}$ through the edge $e_{i, j}$.

\section{Particle Filter Implementation}

For a PF implementation of the Bayesian filter, the posterior filtered density $\mathrm{p}\left(\mathbf{x}\left(t_{0: k}\right), \mathbf{M}\left(t_{0: k}\right) \mid \mathbf{z}\left(t_{0: k}\right)\right)$ of (3) can be approximated by importance samples as

$$
\begin{aligned}
& \mathrm{p}\left(\mathbf{x}\left(t_{0: k}\right), \mathbf{M}\left(t_{0: k}\right) \mid \mathbf{z}\left(t_{0: k}\right)\right) \\
& \propto \sum_{j=1}^{N_{s}} w^{(j)}\left(t_{k}\right) \delta\left(\mathbf{x}\left(t_{k}\right)-\mathbf{x}^{(j)}\left(t_{k}\right)\right)
\end{aligned}
$$

where $N_{s}$ defines the number of particles and the weight

$$
w^{(j)}\left(t_{k}\right) \propto w^{(j)}\left(t_{k-1}\right) \cdot w_{\mathrm{CS}}^{(j)}\left(t_{k}\right) \cdot w_{\mathrm{M}}^{(j)}\left(t_{k}\right) .
$$

where the term $w_{\mathrm{CS}}^{(j)}\left(t_{k}\right)$ defines the weight for the $j$-th particle at time instant $t_{k}$ of Channel-SLAM, see Section III-A and [1]-[4]. To obtain the weight $w_{\mathrm{M}}^{(j)}\left(t_{k}\right)$, the transition map is learned by particle $j$ by counting each transition it makes from $\mathbf{x}_{u}^{(j)}\left(t_{k-1}\right)$ to $\mathbf{x}_{u}^{(j)}\left(t_{k}\right)$ across edge $\tilde{e}$ and its local map for hexagon $H_{\tilde{i}}$. Hence, each particle stores its whole path through the hexagon grid. As mentioned in [17]-[19], learning the map is based on Bayesian learning of multinomial and binomial distributions, where the weight of each particle $j$ can be calculated by

$$
w_{\mathrm{M}}^{(j)}\left(t_{k}\right)=\left\{\frac{N_{\tilde{i}}^{\tilde{e}}+\alpha_{\tilde{i}}^{\tilde{e}}}{N_{\tilde{i}}+\alpha_{\tilde{i}}}\right\} .
$$

The term $N_{\tilde{i}}^{\tilde{e}}$ represents the number of transitions for edge $\tilde{e}$ of hexagon $H_{\tilde{i}}$ and $N_{\tilde{i}}$ is the sum of all transitions of hexagon $H_{\tilde{i}}$ with $N_{\tilde{i}}=\sum_{e=0}^{5} N_{\tilde{i}}^{e}$ for $t_{k}=t_{0}, \ldots, t_{k}$. The terms $\alpha_{\tilde{i}}^{\tilde{e}}$ and $\alpha_{\tilde{i}}=\sum_{e=0}^{5} \alpha_{\tilde{i}}^{e}$ represents the a-priori knowledge regarding the number of transitions across the edges of $H_{\tilde{i}}$ of particle $j$. If no prior information is available, we choose empirically $\alpha_{\tilde{i}}^{\tilde{e}}=$ 0.8. Additionally, if the particle crossed multiple hexagons, the weight update is performed for all crossed edges.
TABLE I: Channel sounder settings

\begin{tabular}{ll}
\hline Parameter & Value \\
\hline RF center frequency & $1.51 \mathrm{GHz}$ \\
Bandwidth $B$ & $100 \mathrm{MHz}$ \\
Number of sub-carriers $N$ & 1281 \\
Sub-carrier spacing $\Delta f$ & $78.125 \mathrm{kHz}$ \\
Transmit power & $10 \mathrm{~mW}$ \\
Signal period $T_{p}$ & $12.8 \mu \mathrm{s}$ \\
Measurement rate $T_{g}$ & $1.024 \mathrm{~ms}$ \\
Transmitter antenna & Omni-directional (V-polarized) \\
Receiver antenna & Omni-directional (V-polarized) \\
\hline
\end{tabular}

\section{MEASUREMEnTS}

This section evaluates the derived algorithm based on outdoor channel measurements, in front of an hangar with a fixed physical transmitter and a moving pedestrian as shown in Fig. 5. The measurements were performed using the MEDAV RUSK-DLR broadband channel sounder in single-input single-output (SISO) mode with the measurement parameters as summarized in Table I. As shown in Fig. 6, the moving pedestrian was equipped with the receive antenna and a Xsense IMU [20]. We captured simultaneously the received signal as well as the turn rates of the gyroscope of the IMU. To measure the coordinates we use a tachymeter TPS 1200 from Leica Geosystems AG [21] which is usually applied in land surveying. The tachymeter has an accuracy in cmdomain based on distance and angular measurements. With the tachymeter, we are capable of measuring coordinates of specific locations. As the transmit antenna is kept at fixed positions, it is straightforward to measure the coordinates of the transmit antenna once before each measurement run. On the other side, the receive antenna moves during the measurement. Hence, we mount a prism as shown in Fig. 6 next to the receive antenna on a stick above the moving person to be always in line-of-sight (LoS) condition to the tachymeter. The tachymeter transmits the measured coordinates to the channel sounder which records the CIRs and the coordinates simultaneously. Fig. 7 shows the scenario from top, with the hangar, physical transmitter position, track, starting and end position. The pedestrian was moving on the indicated track for different loops for $556 \mathrm{~s}$ or $265 \mathrm{~m}$, see Fig. 9. After moving $176 \mathrm{~s}, 331 \mathrm{~s}, 467 \mathrm{~s}$ the pedestrian returns to the starting position. During the walk the pedestrian is moving with a constant speed, between $375 \mathrm{~s}$ and $510 \mathrm{~s}$, and between $477 \mathrm{~s}$ and $495 \mathrm{~s}$, the receiver is standing still. Fig. 8 shows the preprocessed integrated turn rates of the gyroscope for the receiver movement. The start and end time when the pedestrian is standing is indicated by the vertical lines. Fig. 10 shows the recorded unprocessed CIRs versus the receiver traveled distance in seconds.

The accuracy of Channel-SLAM relies directly on the accuracy of the CIR estimations of KEST. Fig. 11 shows the 


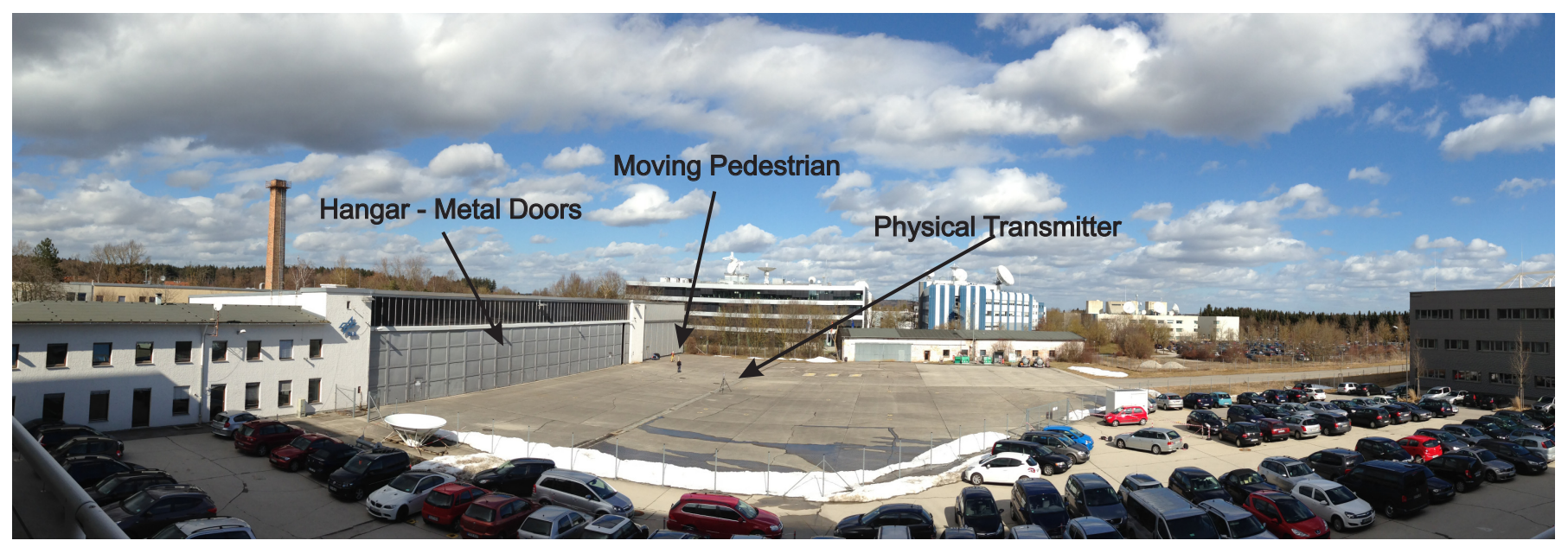

Fig. 5: Measurement scenario: The pedestrian is moving in front of an hangar which is equipped with metal doors. The metal doors acts as a perfect reflecting surface for the transmitted signal.

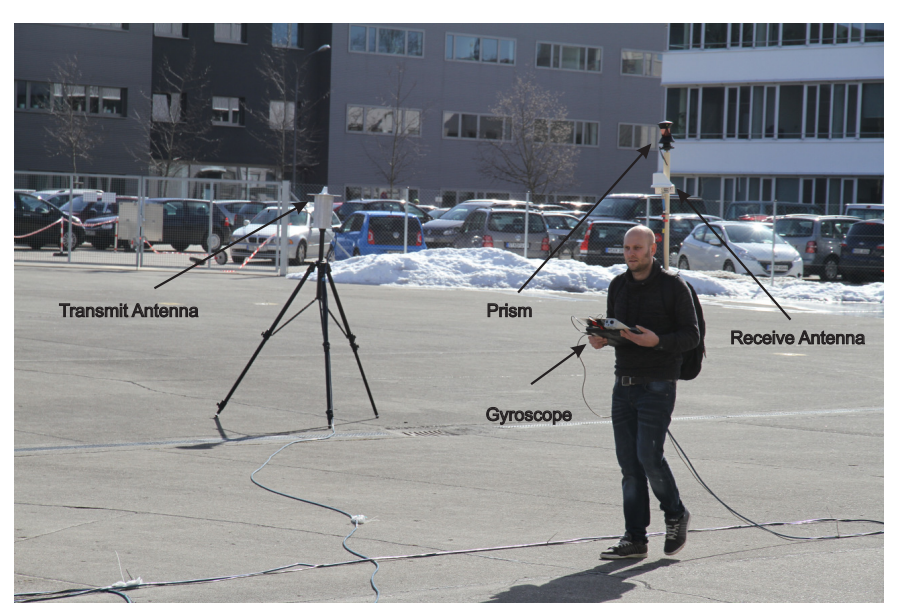

Fig. 6: Moving pedestrian: The receive antenna mounted on a stick next to a prism for measuring the ground truth of the moving pedestrian. The IMU is in the hands of the pedestrian.

estimation results of KEST for the CIR versus the receiver traveled time in seconds. The figure shows long visible paths which can be used by Channel-SLAM for positioning. Fig. 12 shows only the first two tracked paths of KEST which are tracked for the whole receiver movement. The black dotted line indicates the geometrical line-of-sight (GLoS) path, which matches perfectly to the estimation of KEST for the first path. The doors of the hangar are metalized and act as a reflecting surface for the transmitted wireless signal. Hence, we can obtain the position of a VT by mirroring the physical transmitter on the reflecting surface as mentioned in Section II. If we calculate the propagation distance between this VT and the moving pedestrian, we can see that it matches to the estimations of KEST, indicated by the dashed line in Fig. 12. At some time points, the KEST estimations are biased which may come from paths which are close to the reflected path as shown in Fig. 11, e.g. from reflections of the ground.

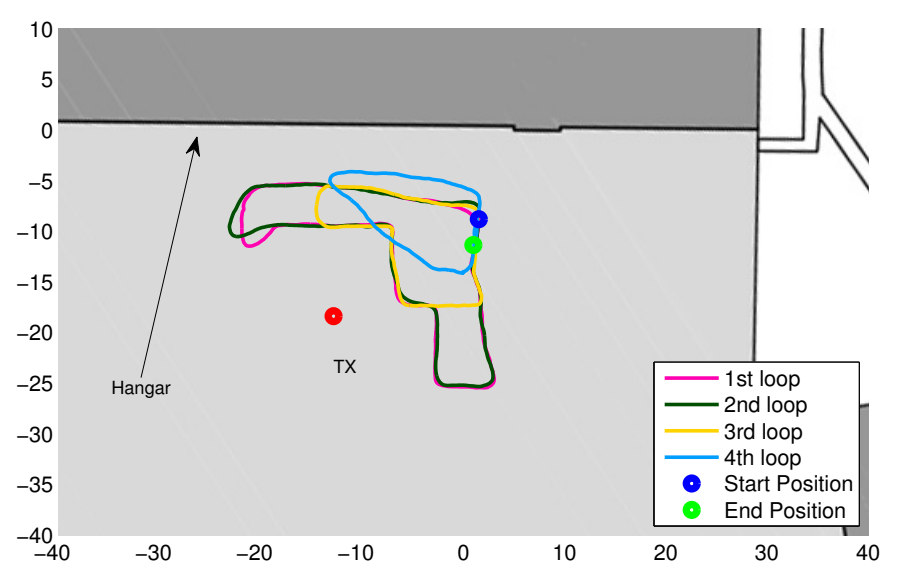

Fig. 7: Measurement scenario with a fixed transmitter and a moving receiver. The receiver moves on the blue track for $556 \mathrm{~s}$ or $265 \mathrm{~m}$, see Fig. 9.

Channel-SLAM does not need any prior information except of a coarse receiver starting position. Hence, the receiver position is initialized in a square with $2 \mathrm{~m} \times 2 \mathrm{~m}$ around the correct starting position. Due to the large number of particles for covering the circular area around the starting position, we use only the two paths of Fig. 12, hence, the LoS path associated to $\mathrm{VT}_{0}$ and the first order reflection associated to $\mathrm{VT}_{1}$ for Channel-SLAM. Here, Channel-SLAM does not assume the knowledge of the transmitter position, the position estimation of $\mathrm{VT}_{0}$ has to be estimated to the physical transmitter position. Additionally, the position estimation of $\mathrm{VT}_{1}$ has to be estimated to the mirrored position of the physical transmitter at the hangar. Fig. 13, Fig. 14, Fig. 15 and Fig. 16 illustrate the performance of the derived algorithm. The left sub-figures show the estimated CIR of Fig. 12 where the vertical dashed line indicate the current time step. The subfigures in the middle show the estimation of Channel-SLAM: estimation of $\mathrm{VT}_{0}$ in blue, estimation of $\mathrm{VT}_{1}$ in orange, 


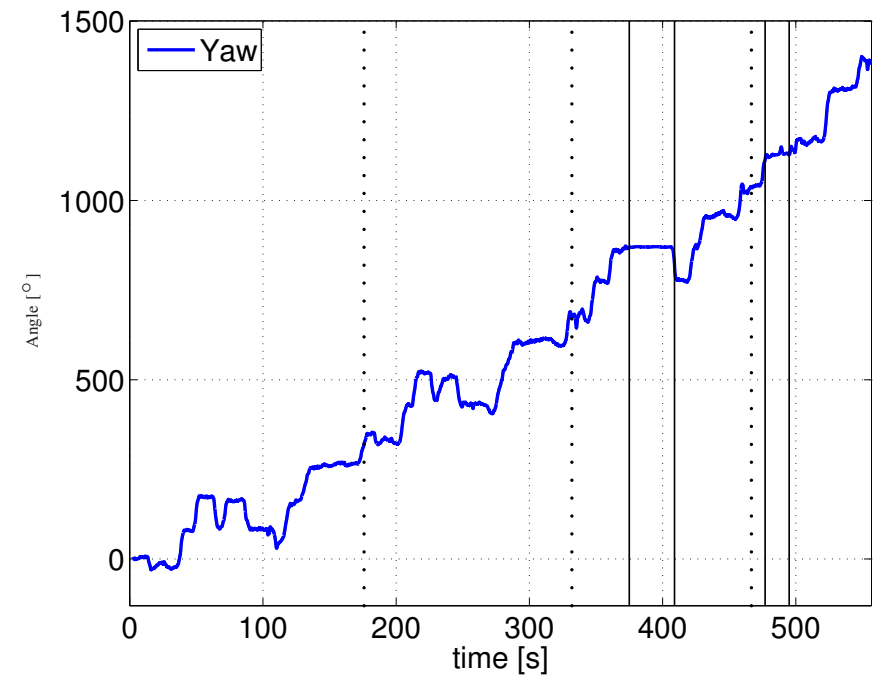

Fig. 8: Integrated turn rates of the gyroscope for the receiver traveled distance. The vertical lines indicate the time periods when the pedestrian is standing. The vertical dashed line indicates when the receiver is returning to the starting position.

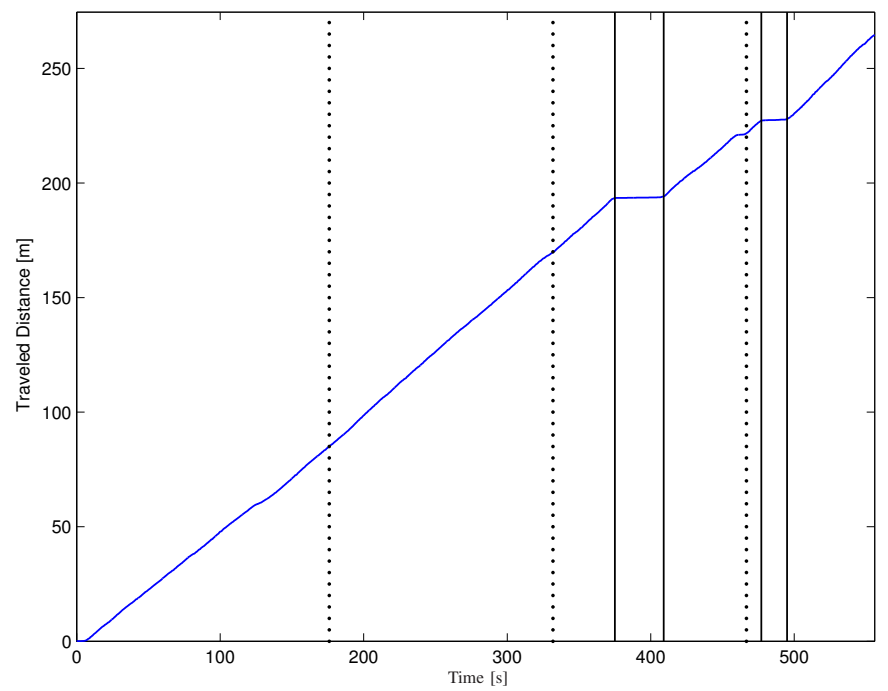

Fig. 9: Traveled distance in meter versus time in seconds. The vertical lines indicate the time periods when the pedestrian is standing. The vertical dashed line indicates when the receiver is returning to the starting position.

mapping of the hexagons where the red hexagons indicate the mapped path with the highest weight. The right subfigures show a more detailed zoomed version of the mapping. Additionally, the middle and right sub-figures indicate by the magenta crosses the ground truth, by the magenta arrows the yaw measurements as shown in Fig. 8, by the green circles the PF estimations of the receiver position and by the red plus the minimum mean square estimate of the receiver position. Fig. 13 shows the initialization at $t_{k}=0 \mathrm{~s}$ : the middle subfigure shows initialization of the VTs of Channel-SLAM. However, the initialization of of $\mathrm{VT}_{0}$ are not visible in this

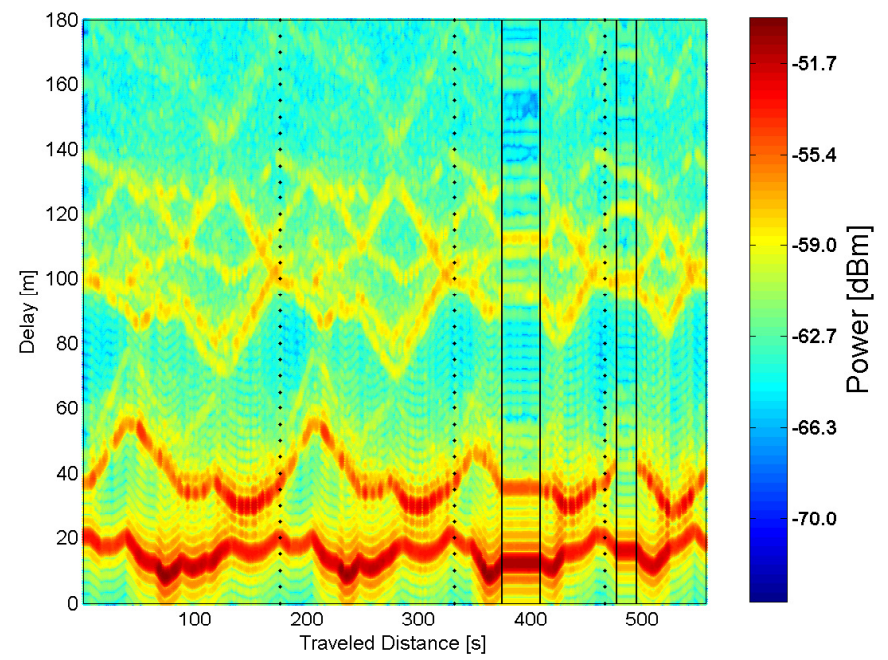

Fig. 10: Recorded unprocessed CIRs versus the receiver traveled distance in seconds.

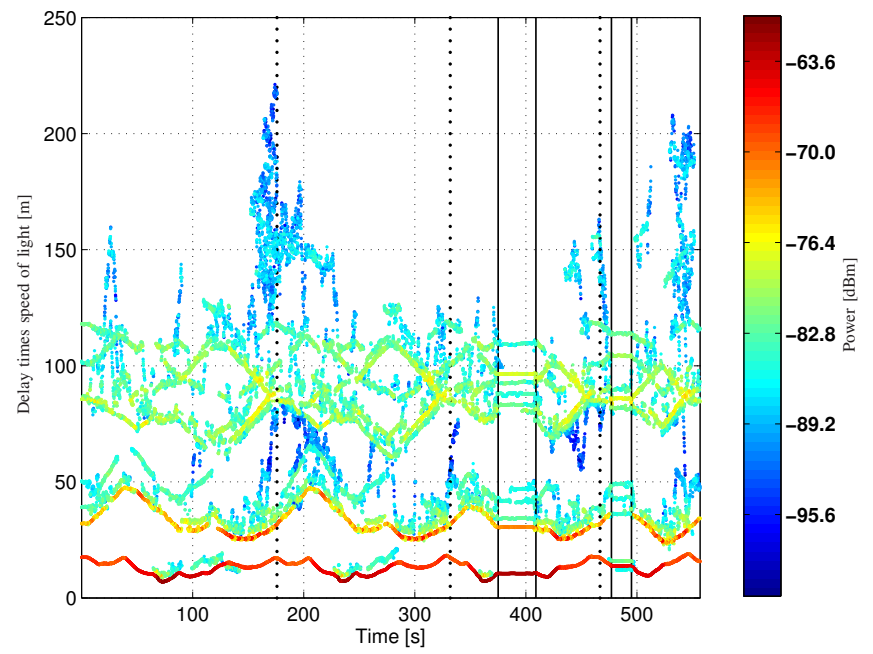

Fig. 11: Estimation results of KEST for the CIR versus the receiver traveled distance in meters. The vertical lines indicate the time periods when the pedestrian is standing and the vertical dashed line indicates when the receiver is returning to the starting position.

figure. Channel-SLAM has no prior information about the physical transmitter position and the VTs positions. According to the first delay measurements, all possible VT positions are initialized in a grid around the starting position. Hence, Channel-SLAM initializes the states for the VT position in a grid with spacing of $0.5 \mathrm{~m}$ in the circular area around the starting position with the radius of the estimated delay. Similarly to Fig. 13, Fig. 14 shows the estimation results after a receiver traveled time of $t_{k}=100 \mathrm{~s}$. Because of the arbitrary receiver movement, the $\mathrm{PF}$ estimations for the locations of the $\mathrm{VT}_{0}$ and $\mathrm{VT}_{1}$ are reduced, however, the position estimation of the receiver has still a high variance. As soon as the receiver returns to the starting position, the hexagon map can 


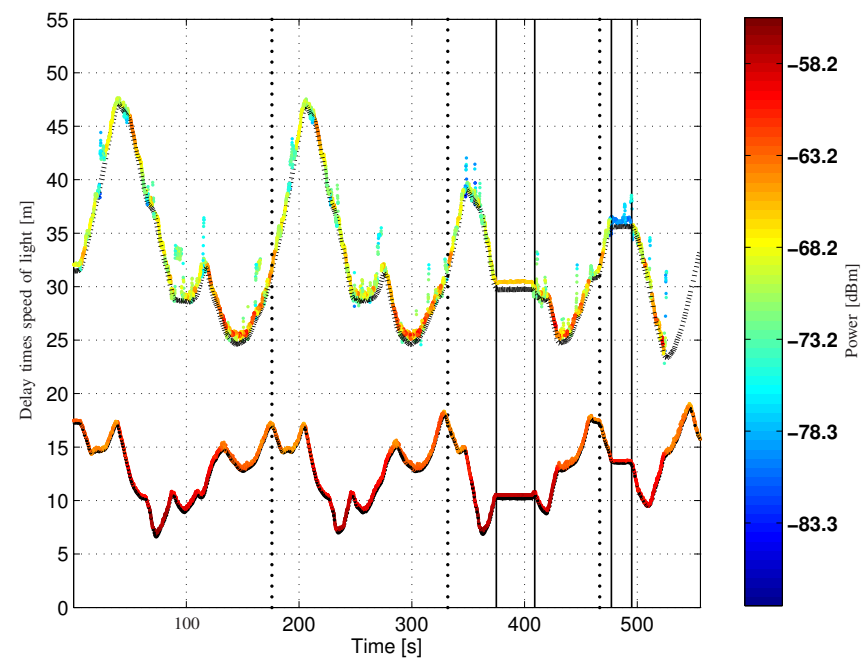

Fig. 12: Estimation results of KEST for the CIR versus the receiver traveled distance in meters. Only the LoS path and the reflected path are visualized. The black dotted line indicates the GLoS path and the black dashed line indicates the calculated propagation distance between VT and the receiver for the receiver traveled distance. The vertical lines indicate the time period when the pedestrian is standing.

be reused as shown in Fig. 15 where the receiver traveled for $t_{k}=200 \mathrm{~s}$. Hence, the uncertainty of the position estimation of the receiver is reduced. Fig. 16 shows the estimation results at the end of the track. We can observe, that we are able to map the receiver path accurately.

\section{CONCLUSIONS}

In this paper, we presented and extended the work on multipath assisted positioning, called Channel-SLAM. The new positioning method uses a SLAM approach to map the receiver position. We propose a probabilistic map that represents the receiver motion in a two-dimensional hexagonal grid. Hence, as soon as the receiver returns to an already mapped position, information of this position can be reused to obtain better position estimations of the receiver as well as the VT positions. We evaluated the algorithm based on measurements with a moving pedestrian and one fixed transmitter.

\section{ACKNOWLEDGEMENT}

This work has been performed in the framework of the DLR project Dependable Navigation and the European Unions Horizon 2020 research and innovation programme under grant agreement No. 636537 HIGHTS (High precision positioning for Cooperative-ITS applications).

\section{REFERENCES}

[1] C. Gentner and T. Jost, "Indoor Positioning using Time Difference of Arrival between Multipath Components," Montbeliard, France, Oct. 2013.

[2] C. Gentner, T. Jost, and A. Dammann, "Accurate Indoor Positioning using Multipath Components," Nashville, TN, USA, Sep. 2013.
[3] C. Gentner, R. Pöhlmann, T. Jost, and A. Dammann, "Multipath Assisted Positioning using a Single Antenna with Angle of Arrival Estimations," Tampa, FL, USA, Sep. 2014.

[4] C. Gentner, R. P. Markus, Ulmschneider, T. Jost, and A. Dammann, "Multipath Assisted Positioning for Pedestrians," Tampa, FL, USA, Sep. 2015.

[5] Y. Shen and M. Win, "On the Use of Multipath Geometry for Wideband Cooperative Localization,” Dec. 2009, pp. 1-6.

[6] M. Froehle, E. Leitinger, P. Meissner, and K. Witrisal, "Cooperative Multipath-Assisted Indoor Navigation and Tracking (Co-MINT) using UWB Signals," Jun. 2013, pp. 16-21.

[7] P. Meissner, K. Witrisal, and K.Witrisal, "UWB for Robust Indoor Tracking: Weighting of Multipath Components for Efficient Estimation," IEEE Wireless Communications Letters, vol. 3, no. 5, pp. 501-504, Oct. 2014.

[8] K. Witrisal and P. Meissner, "Performance bounds for multipath-assisted indoor navigation and tracking (MINT)," Jun. 2012, pp. 4321-4325.

[9] T. Jost, W. Wang, U.-C. Fiebig, and F. Pérez-Fontán, "Detection and Tracking of Mobile Propagation Channel Paths," IEEE Trans. Antennas Propag., vol. 60, no. 10, pp. 4875-4883, Oct. 2012.

[10] R. Smith and P. Cheeseman, "On the Representation and Estimation of Spatial Uncertainty,” vol. 5, no. 4, pp. 56-68, 1986.

[11] T. Jost, W. Wang, U.-C. Fiebig, and F. Pérez-Fontán, "A Satellite-toIndoor Channel Model: Multipath Components," Prague, Czech Rep., Mar. 2012.

[12] M. Arulampalam, S. Maskell, N. Gordon, and T. Clapp, "A Tutorial on Particle Filters for Online Nonlinear/Non-Gaussian Bayesian Tracking," IEEE Trans. Signal Process., vol. 50, no. 2, pp. 174-188, Feb. 2002.

[13] D. Titterton and J. Weston, Strapdown Inertial Navigation Technology, 2nd ed. The American Institute of Aeronautics and Astronautics, 2004.

[14] O. J. Woodman, C. O. J. Woodman, and O. J. Woodman, “An Introduction to Inertial Navigation," 2007.

[15] B. W. Parkinson and J. J. Spilker Jr., Global Positioning System: Theory and Applications, Vol. 1. American Institute of Aeronautics and Astronautics Inc., 1996.

[16] B. Ristic, S. Arulampalam, and N. Gordon, Beyond the Kalman Filter: Particle Filters for Tracking Applications. Artech House, 2004.

[17] P. Robertson, M. Angermann, and B. Krach, "Simultaneous Localization and Mapping for Pedestrians using only FootMounted Inertial Sensors," in In Proc. UbiComp 2009, ACM, 2009, pp. 93-96.

[18] M. Angermann and P. Robertson, "FootSLAM: Pedestrian Simultaneous Localization and Mapping Without Exteroceptive Sensors - Hitchhiking on Human Perception and Cognition," Proceedings of the IEEE, vol. 100, no. Special Centennial Issue, pp. 1840-1848, May 2012.

[19] M. Puyol, D. Bobkov, P. Robertson, and T. Jost, "Pedestrian Simultaneous Localization and Mapping in Multistory Buildings Using Inertial Sensors," Intelligent Transportation Systems, IEEE Transactions on, vol. 15, no. 4, pp. 1714-1727, Aug 2014.

[20] "Xsens MTx IMU," Tech. Rep. [Online]. Available: http://www.xsens.com/en/general/mtx

[21] Leica tachymeter geosystems. http://www.leicageosystems.com/en/index.htm. 

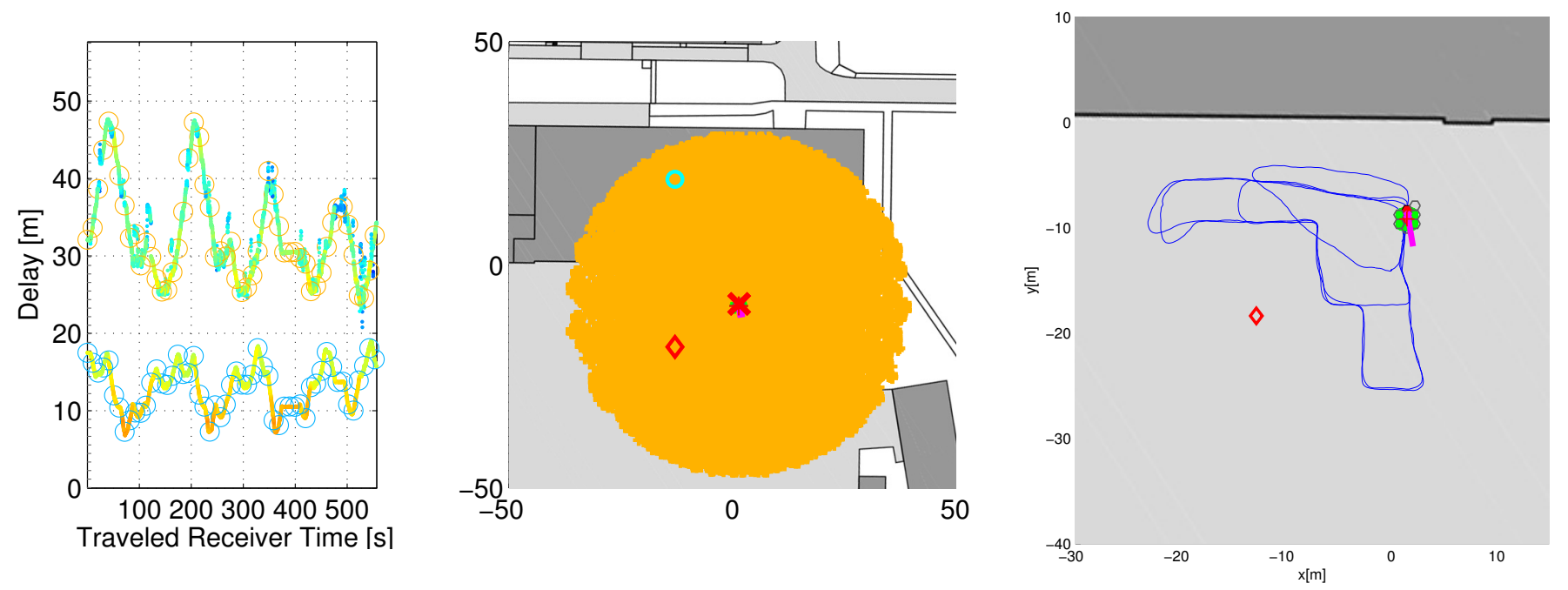

Fig. 13: Initialization at $t_{k}=0$. The left figure shows initialization of the VTs of Channel-SLAM: in blue the PF estimation of $\mathrm{VT}_{0}$ (not visible) and in orange the PF estimation of $\mathrm{VT}_{1}$. The right figure shows the initialization of the hexagons. The magenta cross indicates the ground truth and the magenta arrow indicates the yaw measurements.
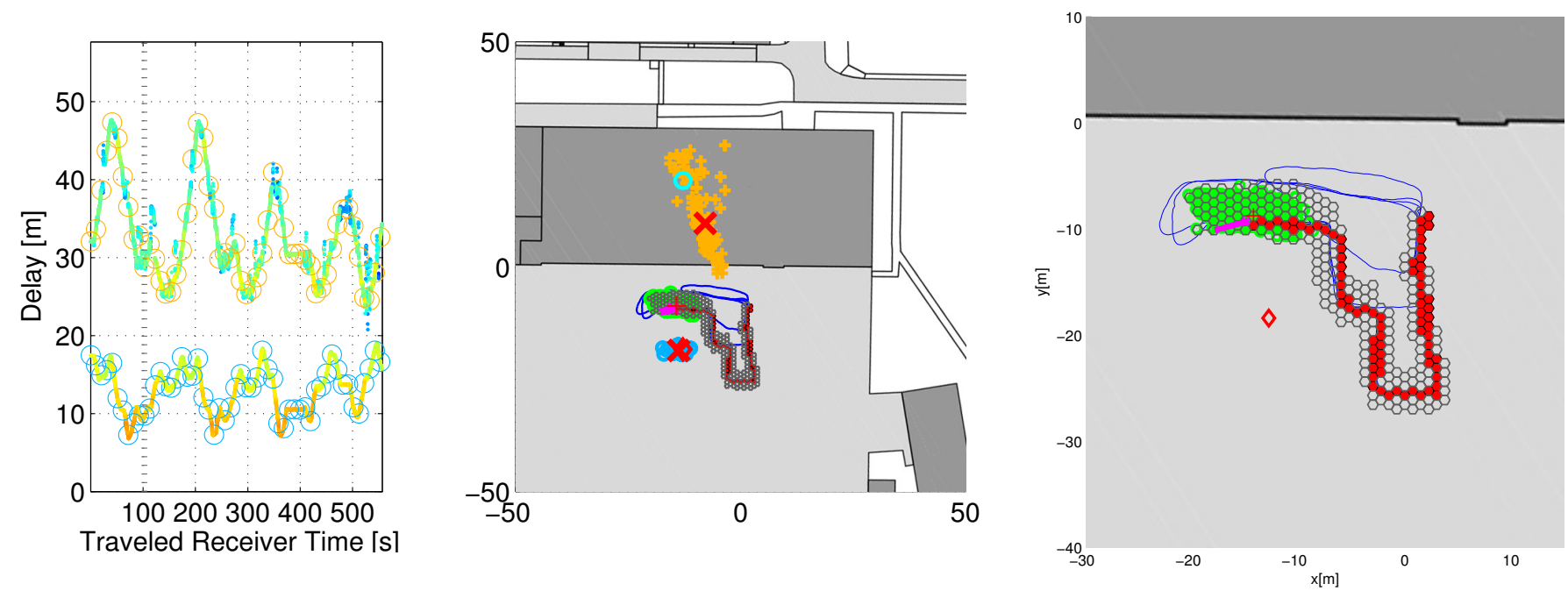

Fig. 14: Receiver traveled time $t_{k}=100 \mathrm{~s}$. The left figure shows the VTs of Channel-SLAM: in blue the PF estimation of $\mathrm{VT}_{1}$ and in orange the PF estimation of $\mathrm{VT}_{1}$. The red hexagons show the mapped track with the highest weight. 

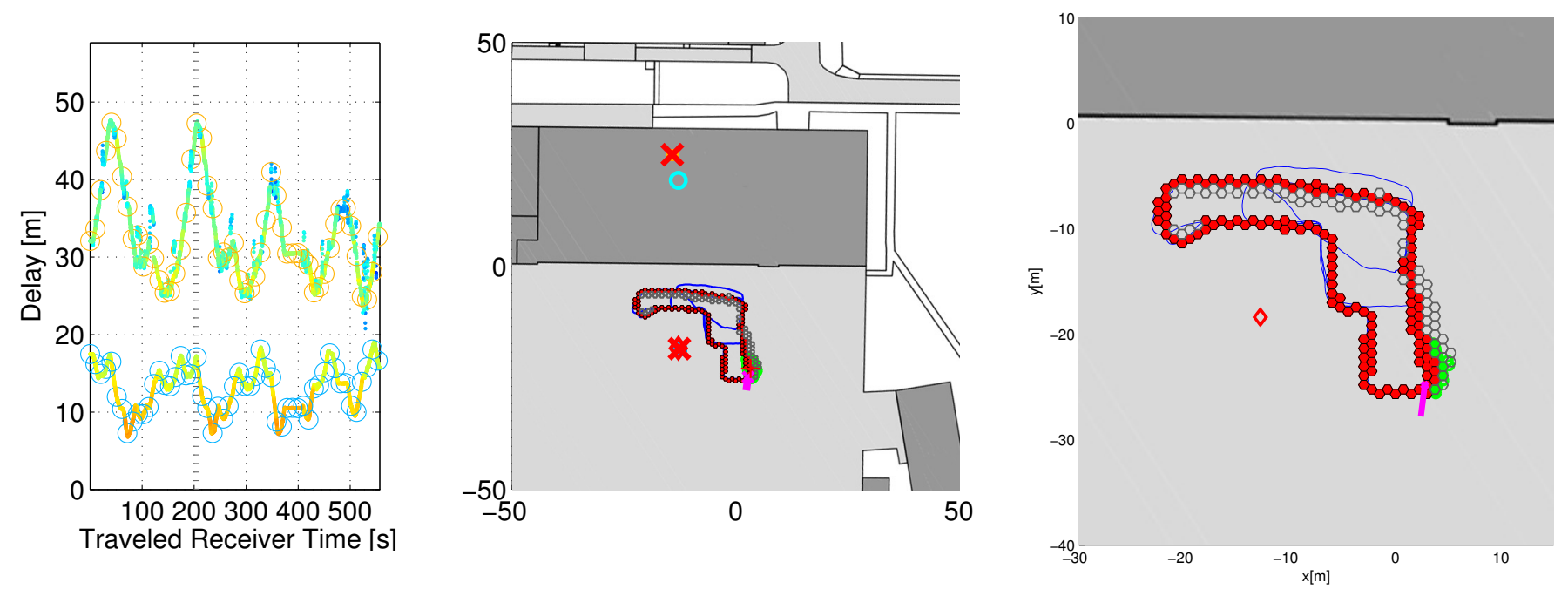

Fig. 15: Receiver traveled time $t_{k}=200 \mathrm{~s}$. The left figure shows that the estimation of the VTs of Channel-SLAM converged to the correct VT positions. The algorithm reuses the mapped location of the first loop.
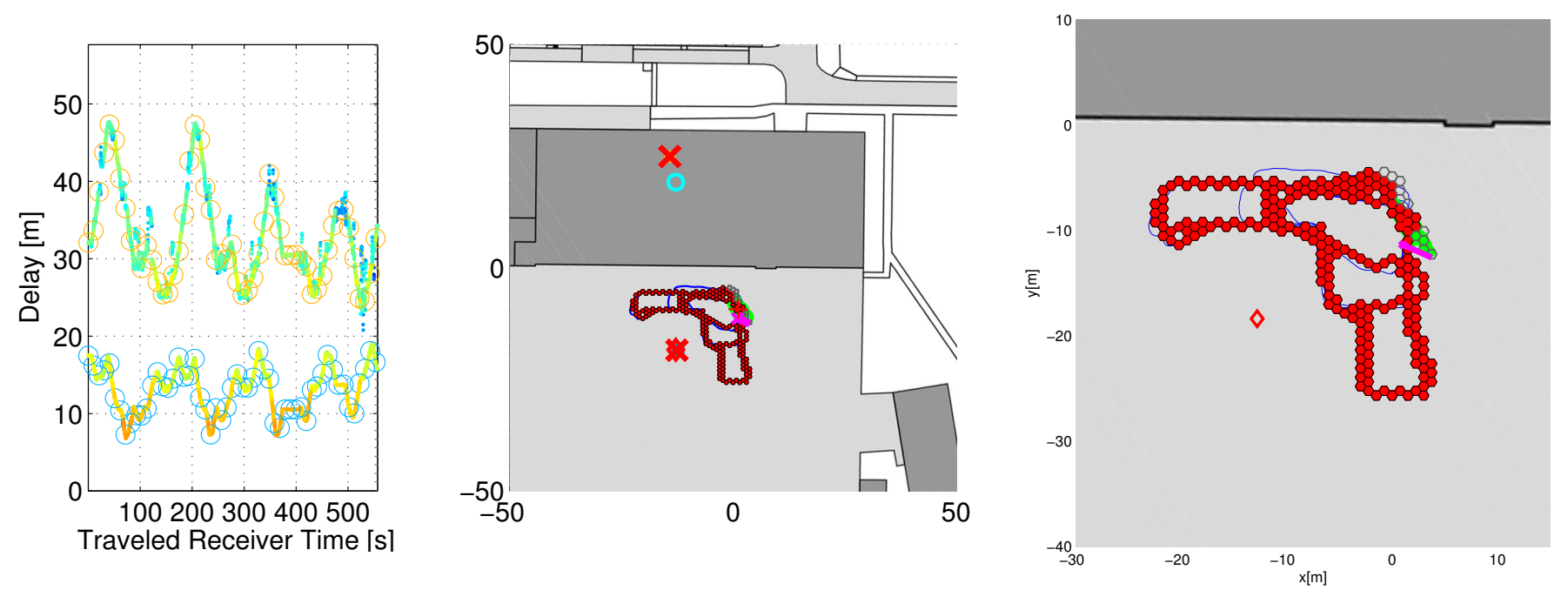

Fig. 16: Receiver traveled time $t_{k}=556 \mathrm{~s}$, end of the track. The left figure shows that the estimation of the VTs of ChannelSLAM converged to the correct VT positions. We can observe by the magenta arrow that the heading was drifting by $50^{\circ}$, however, Channel-SLAM was not influenced. 